\title{
Literarische Mehrsprachigkeit und Transkulturalität. Der Dialog zwischen den Kulturen und das Echo von Mimikry und sprachlicher Hybridität im Werk deutsch-türkischer Autorin- nen
}

\author{
Maria E. Brunner \\ Pädagogische Hochschule Schwäbisch Gmünd
}

The beginnings of migration literature in Germany were noticeable 20 years after the first agreements for work enlistment in the 1950s between countries around the Mediterranean on the one hand and Germany on the other. Well into the 1980s migration literature was considered to be a politically motivated, autobiographical and experiential kind of literature produced by migrants, most of whom were initially foreign workers. Today migrant/migration literature or intercultural literature is considered to be the work of a generation of authors originating from families with migration experience, or migrants themselves. It is the linguistic hybridity and thematic alterity in these works which form the pre-condition for the dialogue between cultures and languages; a dialogue initiated by the successful examples of migration literature currently available on the market. Indeed, a considerable number of German-Turkish authors' works enrich the cultural and literary scene in Germany. The article analyses the use of hybridity and mimicry as central themes in the novel Der Mond isst die Sterne auf (1998) by Dilek Zapctioglu. This novel is then compared with the stories in the collection Mutterzunge (1998) and with a novel by Emine S. Özdamar, more specifically with the novel Die Brücke vom goldenen Horn (1998), exploring language and thematic hybridization. Central themes in migration literature are issues such as the loss of the homeland, culture shock, alienation, life in and between two languages and cultures, as well as everyday, subliminal discrimination and the accumulating communication problems. The novels can therefore be defined thematically on the basis of their changing time and space coordinates, their shared theme of space diversity, their oscillation between the homeland and the foreign land, the linguistic diversity of their voices and the reflection they offer on the diaspora.

\section{Einleitung}

Migrationsliteratur ist inzwischen unübersehbar in der deutschen Literaturszene präsent und weist "eine gewisse Selbstständigkeit auf"; die Autorinnen wollen vor allem als "Künstler/innen anerkannt werden, während die multikulturelle Industriegesellschaft in ihnen genuine Kulturvermittler sieht" (Sölcün 2000: 135). Der Begriff meint alle fiktionalen Texte, die geprägt wurden durch die eigene Migration der Autoren und Autorinnen oder durch 
die Migration ihrer Eltern sowie durch einen Sprachenwechsel (die meisten Autoren/innen der zweiten Migrantengeneration schreiben inzwischen in deutscher Sprache). ${ }^{1}$ Die "Literatur der türkischen Minderheit" erscheint bei renommierten deutschen Literaturverlagen und auf deutschen Bestsellerlisten (wie Pirinçcis Krimi Felidae). Türkische Namen tauchen unter den Gewinnern angesehener Literaturpreise auf, etwa Zehra Çirak (Hölderlin Preis für Lyrik 1994) und Emine Sevgi Özdamar (Bachmann Preis 1991).

In den 70er-Jahren setzte in der BRD die literarische Produktion von Autorinnen aus anderen Sprach- und Kulturräumen ein (beginnend mit italienischen Migrantenautoren) - also erst 20 Jahre nach den ersten Anwerbeabkommen. Inzwischen werden "Zweisprachige Schriftsteller/innen in Deutschland" in neueren Schulbüchern für den Deutschunterricht an der Oberstufe zum Kanon der deutschsprachigen Literatur nach 1945 gezählt (Biermann \& Schurf: 360-365). ${ }^{2}$ Zweisprachige Schrifsteller/innen untergraben so die tradierte Vorstellung von Literaturgeschichte als Nationalgeschichte, denn ihre Werke entstehen an den Rändern der Nationen und problematisieren dadurch nationale Identität und kulturelle Homogenität. Mit dem Sprachenwechsel der Autoren/innen ist auch die Frage nach der Standortbestimmung dieser Literatur verbunden: Weinrich (1984: 22) hatte sie als "Literatur der Betroffenheit" und sogar als "deutsch", Ackermann (1985: 55) als "Ausländerliteratur" bezeichnet und in ihr eine "Bereicherung" für die deutsche Literatur gesehen. Amodeo (1996: 94) spricht von Literatur "ausländischer Autoren", Weigel von "Minderheitenliteratur". Adelson (1991: 68) kritisiert gerade den "Bereicherungstopos" als ethnozentrisch, da trotz dieser "ausländischen" Zusätze das Dogma vom autochthonen deutschen Kern der deutschsprachigen Gegenwartsliteratur nicht hinterfragt werde; sie verwahrt sich gegen Ausgrenzungsbestrebungen (zwischen einer "deutschen" und einer "ausländischen” Literatur). Weigel (1992: 214) verwendet den Begriff "Literatur in der Fremde". Doch auch damit werden pauschale geografische und politisch-soziale Zuordnungen vorgenommen. T. Wägenbaur (1995: 22) spricht von "interkultureller Literatur", damit die Autoren nicht der "einen oder anderen sozialen Gruppe zugeschlagen werden". Wierschke (1994: 33) definiert sie als "kleine Literatur" in einer "Nische" des deutschen Literaturbetriebs. Einige Autoren/innen (unter anderem Zehra Çirak und Güney Dal) wehren sich gegen den tribalistischen Begriff “Ausländerliteratur”. Im Folgenden werden zwei Romane aus der zeitgenössischen deutsch-türkischen Literatur vorgestellt, geschrieben von Autorinnen, deren Identität durch die eigene Migration oder durch die Migration ihrer Eltern (etwa in Form von Binnenmigrationen in der Türkei bei Özdamar) mit geprägt wurde. Die Autorinnen Dilek Zaptcioglu und Emine Sevgi Özdamar haben in ihren Werken darauf hingewiesen, dass die Begegnung mit der Fremde/dem Fremden/den Fremden die Frage nach der eigenen Identität/den eigenen Identitäten in besonderer Weise aufwirft. Sie müssen sich unter anderem mit den Rollenzuweisungen als Fremde auseinander setzen, mit den Erfahrungen der Migrationssituation, wobei sie zu unterschiedlichen Ergebnissen gelangen. Sprachenwechsel ist als herausra- 
gendes Merkmal der jüngeren Autoren/innen anzusehen, die Migration “inhaltlich und ästhetisch gestalten” (Rösch 1992: 37). Nach Howard werden

Folgen des Übergangs in eine fremde Situation gezeigt, in der das erzählte Subjekt sich und die Welt in veränderter Sicht wahrnimmt [...]. Es geht vor allem um die Lage des Menschen ohne eigenen Raum und um Erkenntnisse, die durch Verlust und Suche, durch den Wegfall einer vertrauten Sphäre und das Bemühen um die wie auch immer geartete Gewinnung neuen Bodens hervorgerufen werden. (Howard 1997: 11, 12)

Howard lehnt die Begriffe Migrations- oder Migrantenliteratur ab, da sich diese Literatur nicht nur an Migranten richtet, nicht nur von solchen produziert wird und nicht nur von Migration handelt. Die lexikalische Komposition bedingt die Ambiguität dieser beiden Begriffe: dem ersten Glied dieser Wortbildungen können die Kasusrollen Agens, Rezipient und Patiens "ohne grammatische oder semantische Restriktion" zufallen (Howard 1997: 10).

In der "Gastarbeiterliteratur" aus den 60er-Jahren und den frühen 70er-Jahren wurde die Situation des eingewanderten Arbeiters, der Verlust von Heimat und Familie, die Orientierungslosigkeit und die Isolation in der Fremde beschrieben. Der Begriff "Gastarbeiterliteratur" war nicht immer zutreffend, da Autoren wie Pazarkaya, Baykurt und Akcam sowie Aysel Özakin schon in der Türkei als Autoren tätig waren. Inzwischen sind Begriffe wie Ausländer- oder Gastarbeiterliteratur obsolet geworden (sie entsprechen nicht mehr dem Selbstverständnis der jungen Generation), weil es mittlerweile Autoren/innen gibt, deren Eltern zwar aus dem Ausland kamen, sie aber selbst in Deutschland geboren sind oder bereits in frühester Kindheit hierher kamen und inzwischen die deutsche Staatsbürgerschaft erworben haben.

Die Autoren/innen von Migrations- oder Migrantenliteratur definieren sich heute eher als über- oder transnational "im Sinne der neuen kulturhistorischen und ethnologischen Perspektiven auf die Literatur von ethnischen Minderheiten in multikulturellen Gesellschaften" (Esselborn 1997: 70). Ihre Werke können beitragen zur Bewusstmachung von Stereotypen, der Ausgrenzung von Migranten sowie der Rechtsunsicherheit von "Illegalen" und sie halten den Autochthonen den Spiegel vor.

Thematisch lassen sich die Werke der Migrations- oder Migrantenliteratur abgrenzen durch ihre Mehrsprachigkeit, den Wechsel von Zeit- und Raumkoordinaten, das Thema der Ortsvielfalt, die Oszillation zwischen Heimat und Fremde und durch die Reflexion der Diaspora; in neueren Texten liegt keine eindeutige Themeneingrenzung mehr vor. Insofern stimmt Wägenbaurs These, dass das "erste Problem, über dieses neue Genre zu sprechen, [...] seine Bezeichnung" ist, immer noch (Wägenbaur 1995: 22).

H. Bhabha (1996: 345) zufolge entspringen postkoloniale Perspektiven nicht nur dem Kolonialerbe der Dritten Welt, sondern ebenso den Diskursen der Minderheiten allgemein, denn die Erfahrung gesellschaft- 
licher Marginalität nimmt gerade in nicht kanonischen Formen der Kultur Gestalt an. Diese gesellschaftliche Marginalisierung zwingt uns nach Bhabha zur "Auseinandersetzung mit einem Kulturbegriff jenseits von Kunstwerken oder einer normativen Ästhetik". In seinen Augen ist Kultur sowohl transnational wie "translational" ("verschoben, übersetzend"). Denn solche räumlichen Verschiebungen gehen heute Hand in Hand mit transnationalen Dimensionen der kulturellen Umbrüche: "Die postkoloniale Perspektive zwingt uns dazu, die grundsätzlichen Beschränkungen des konsensorientierten [...] Verständnisses kultureller Gemeinschaft zu überdenken", denn sie behauptet, "kulturelle und politische Identität werde mittels eines Alteritätsprozesses konstruiert” (Bhabha 1996: 347).

Die Werke von zwei deutsch-türkischen Autorinnen werden im Folgenden als literarische Ausdrucksformen von Mimikry, Alterität und Hybridität präsentiert. Die Schreibansätze von Zaptcioglu und Özdamar werden als Ausdruck eines Dezentralisierungsprozesses verstanden, der das Standard-Hochdeutsch um neue varietätenspezifische Stilisierungen ergänzt und durch die literarische Relativierung individueller wie kollektiver kultureller Identität neue Ausdrucksformen der Hybridität entstehen lässt.

\section{Mimikry und Hybridität bei Dilek Zaptcioglu}

In Der Mond isst die Sterne auf von Dilek Zaptcioglu geht es um die Identitätsfindung von Jugendlichen mit Migrationshintergrund in Berlin nach dem Fall der Mauer. Die völlige Anpassung (Assimilation) des Kolonialisierten wird am Beispiel von Ömers Freund Kenan vorgeführt:

\footnotetext{
[...] wenn man Kenan überhaupt als Türken bezeichnen wollte. Seine Mutter war Deutsche und sein Vater hatte mit den Türken, die ich kannte, nicht viel gemeinsam, denn er war Arzt. Wenn es stimmte was die Leute behaupteten, waren Türken in seiner Praxis nicht gern gesehen, er unterhielt sich mit ihnen ausschließlich auf Deutsch und ich war selbst Zeuge davon geworden, dass er nicht einmal zu Hause Türkisch sprach. (Zaptcioglu 1998: 41)
}

Die begrenzte Anpassung wird hingegen an Ömers Bruder Adnan gezeigt; er ist in die Rolle des Italieners geschlüpft und betreibt ein florierendes italienisches Restaurant in Westberlin. Aufgrund der daraus resultierenden "Mimikry" erscheint die koloniale Autorität gebrochen: der begrenzt Angepasste stellt nämlich zugleich eine verzerrte Spiegelung der Diskurse der Mehrheitsgesellschaft dar (nur ein erfolgreicher Gigolo und Kleinunternehmer erreicht eine gewisse gesellschaftliche Reputation). Adnan steht als Produkt einer zivilisatorischen Mission in einem ironischen Verhältnis zu seinen Gästen und kann diese "Mimikry" (als Medium der Befreiung) auf subversive Weise gegen die Mehrheitsgesellschaft einsetzen. Bhabha (1996: 353) hat mit den Begriffen "Nachahmung, Hybridität und falsche Höflichkeit" dargestellt, wie "dieser Schwellenmoment der Identifikation eine subversive Strategie subalternen Handlungsspielraums hervorbringt". 
Doch nachdem Adnan als "'Der Sohn des Opfers"” bei der Kölner Protestkundgebung gegen Ausländerfeindlichkeit (auf Grund des noch nicht restlos aufgeklärten Unfalls seines Vaters) im Fernsehen präsentiert wird, kann er diese Mimikry nicht mehr durchhalten: “Aus mit dir, Adnan, dachte ich im Stillen, jetzt, wo die ganze Nation dich auf dem Bildschirm hat, wirst du dich nicht mehr als Prego, signorina ausgeben können. Wenigstens etwas, das meinem Vater gefallen wird" (Zaptcioglu 1998: 207). Adnans Mimikry erweist sich hier als eine auf Imitation beschränkte Schutzanpassung und eine bewusste Täuschung, aber zugleich auch als "Zeichen des Un(an)geeigneten (inappropriate), eine Differenz oder Widerspenstigkeit" (Bhabha 2000: 127). Bhabha stuft Mimikry als eine der am schwersten zu fassenden und gleichzeitig effektivsten Strategien ein, denn sie entsteht als die Repräsentation einer "Differenz" (Bhabha 2000: 132), die ihrerseits ein Prozess der Verleugnung ist; Mimikry "verbirgt keine Präsenz oder Identität hinter ihrer Maske" (Bhabha 2000: 130); dabei beruft er sich auf E. Said und definiert Mimikry als “ironischen Kompromiß” (Bhabha 2000: 126). Hier sind auf Grund ihrer gesellschaftlichen Akzeptanz die Italiener das koloniale Subjekt und deren mimetische Nachahmung durch das koloniale Objekt (den Türken Adnan) markiert zugleich eine "Ambivalenz der Mimikry (beinahe dasselbe, aber nicht ganz)" (Bhabha 2000: 127). Gerade diese besondere Form der "Posse" (Bhabha 2000: 127) macht nach Bhabha die Mimikry aus. Adnan liefert ein solches Beispiel „für koloniale Imitation“ (Bhabha 2000: 127), denn er gibt sich als "Adriano" aus und umwirbt seine autochthonen Gäste mit italienischem Charme:

'Prego, Signorina!' sagte er zu der Mitvierzigerin ... Mein Bruder lächelte sie höflich an und sagte: 'In Napoli sind alle Frauen Signorinas [....]' 'Adriano!' sagte ich laut. 'Der Plural von signorina ist nicht signorinas, was für ein Italienisch lernst du eigentlich auf der Volkshochschule?' (Zaptcioglu 1998: 31)

Das dominante Kennzeichen der Mimikry ist also ihre Ambivalenz, denn diese kulturelle Strategie spielt mit vorgegebenen Identitätsmustern (wie Adnan dies tut), indem sie diesen zwar folgt, sie aber nie restlos erfüllt somit bringt Adnan nicht nur die Fiktionalität der Vorgabe zum Vorschein (als Vorzeigeitaliener), sondern auch die Unmöglichkeit, sich dieser gänzlich unterzuordnen. Bhabha betont zwar die Ambivalenz und Instabilität von kolonialer Mimikry, da sie den Ist-Zustand (die koloniale Herrschaft) perpetuiert; doch eine bestimmte Ähnlichkeit zwischen kolonialem Subjekt und Objekt legt den konstruierten Charakter von Identitäten offen und destabilisiert damit (post)koloniale Herrschaft. Bhabha weist der Mimikry eine grundlegende Unterwanderungsfunktion $\mathrm{zu}$; auch in Zapctioglus Roman fungiert Mimikry als intentionale Angleichung bei Beibehaltung einer Differenz, die ein destabilisierendes Potential in sich birgt.

Die Hybridität der Gruppe Jugendlicher mit Migrationshintergrund in Zaptcioglus Roman verweist auf die Auflösung der Dichotomie IdentitätAlterität und bietet den Jugendlichen in ihrem "Bund der Geächteten" die 
Möglichkeit, sich in einem third space oder im in-between zu profilieren. Das emanzipatorische Potential, das ihre These "wir sind alle irgendein Gemisch" (Zaptcioglu 1998: 127) freisetzt, führt darüber hinaus auch zu komplexen Grenzzonenerfahrungen, wodurch soziale, kulturelle und geschlechtsspezifische Gegensätze abgebaut werden. Der identitätstheoretische Ansatzpunkt im Roman von Zaptcioglu beruht nicht auf dem Prinzip des Gegensatzes, sondern auf dem der Differenz; so entstehen ganz andere Kulturmuster als solche, die auf binäre Beschreibungssysteme zurückgehen.

In Abweichung von der eindimensional-linearen Abfolge einer "Geschichte" spaltet sich das plurale Subjekt der Aussage (Ömer, Seyfullah) in ein sprechendes und ein gesprochenes auf. Die Komplexität von Identität und Zugehörigkeit wird bei Zaptcioglu von einer (multikulturell zusammengesetzten) Gruppe von Jugendlichen auch durch das Lesen von Heines "Harzreise" sowie über Heines jüdische Minoritätsidentität verhandelt. Der Unterschied deutsch-nicht deutsch ist im Freundeskreis Ömers obsolet geworden: die migrierende (im Sinne von den Aufbruch wagende) Individualität wird an die Stelle von erstarrter Ethnizität gesetzt; dies mündet in die interkulturelle Utopie: "Mich interessiert, ob wir Freunde sind oder nicht” (Zaptcioglu 1998: 129).

Hybrid ist in Der Mond isst die Sterne auf somit alles, was aus der Vermischung von Traditionen, Diskursen und Zeichen entsteht. Dies zeigt sich an den Erinnerungen Kamils, der lange einer der Arbeitskollegen von Ömers Vater gewesen war: "Verschwenderisch ging er mit Worten um, meistens in Türkisch, mit hineingesprenkelten deutschen Worten wie 'Heim', 'krank gemacht', 'Betriebsrat', 'Arbeitsamt', ich schätze, er wusste gar nicht, wie diese Wörter auf Türkisch hießen” (Zaptcioglu 1998: 148). Dieser Vermischung liegen verschiedene Möglichkeiten und Strategien der Aneignung, Wiederaneignung, Kontextualisierung und Rekontextualisierung zugrunde, wobei auch der Vorgang der Vermischung selbst immer schon als Hybridisierung zu beschreiben ist.

\section{Inszeniertes Sprechen und Hybridität bei Emine S. Özdamar}

Bei Emine S. Özdamar finden sich in den Erzählungen im Band Mutterzunge und im Roman Die Brücke vom Goldenen Horn Etappen türkischer Migration vor allem am Beispiel junger Frauen. Ein zentraler Aspekt des Romans ist im ersten Teil die Darstellung der Unterschiede zwischen türkischen Frauen und Männern bei der Bewältigung der Migrationssituation; türkische Männer bilden eine Rangordnung innerhalb ihrer Gruppen, suchen Schutz in politischen Gruppen und bei den Frauen. Das Bewältigungsverhalten der Frauen unterscheidet sich davon grundlegend, denn bei ihnen dominieren die Suche nach Geborgenheit in der eigenen Gruppe, die Suche nach der Mutter, aber auch die gegenseitige Kontrolle und teilweise die Unterwürfigkeit traditionsorientierter Frauen gegenüber den Männern. Die Männer emigrieren scheinbar ohne bestimmte Lebensziele, die Frauen hin- 
gegen haben ein bestimmtes Ziel. Beide Geschlechter sind allerdings nur in der Gruppe unterwegs, zeigen ein traditionsorientiertes Rollenverhalten und werden verfolgt von der Sehnsucht nach der Heimat.

Özdamars Zugang zur deutschen Sprache hat sich über das Schauspiel entwickelt: "'Ich habe gemerkt, dass die deutsche Sprache meine körperliche Erfahrung ist. Ich könnte fast sagen, die Wörter haben Körper. Ich habe sie entweder selbst gespielt oder sie aus dem Körper der Schauspielerfreunde gehört"” (Özdamar in von Saalfeld 1989: 167). Inszeniertes Sprechen und Mimikry sind hier Formen der Nachahmung, für die die Differenz zentral bleibt; daher kann Mimikry auch bei Özdamar eine Parodie der Mehrheitsgesellschaft und Kritik beinhalten:

Einmal lief ich mit Ibni Abdullah in meinem Körper auf der Straße, es kamen ein Mann und eine Frau, sie sind erst an mir vorbeigegangen, dann haben sie sich in die Augen geguckt und mit ihren Köpfen rechts und links gewackelt. Ich blieb stehen, dann kamen zwei Männer. 'Meine Herren, spielt in meinem Gesicht ein Affe?' 'Nein', haben die zwei Männer gesagt, 'Ihr Gesicht ist sehr normal, aber besser hätten sie das Ehepaar gefragt'. (Özdamar 1998: 21)

Die Ich-Erzählerin ist Orientalin und die deutschen Passanten reagieren auf sie ablehnend, weil sie als Migrantin von der Norm des Deutsch-Seins abweicht; hier benutzt Özdamar als Subtext Gates' Signifying Monkey, wo ebenfalls das rassistische Bild des angeblich affenähnlichen Schwarzen ironisch verkehrt wird.

Fremd wirken die assoziativen Reihungen und Neologismen Özdamars auf uns monokulturell geprägte Leser deshalb, weil wir durch ein ganz anderes historisches Vorwissen, andere gesellschaftliche Entwicklungen, Kulturtraditionen und Primärerfahrungen als die bikulturell geprägte IchErzählerin definiert sind, aber auch aufgrund der unterschiedlichen Sprachstruktur Türkisch-Deutsch; denn der deutsche Leser hat Verfügungsgewalt über seine (Mutter)Sprache, der ausländische Autor ist hingegen bloß Gast in der für ihn fremden Sprache. Durch rhythmisch gleichförmig repetierte Parataxen und Aufzählungen wird die Monotonie der Fabrikarbeit auf der Formebene des Romantextes widergespiegelt: "Jede saß allein am Tisch. Jedes Gesicht schaute auf den Rücken der anderen. Während man arbeitete, vergaß man die Gesichter der anderen. Man sah nur Haare, schöne Haare, müde Haare, alte Haare, junge Haare, gekämmte Haare, ausfallende Haare" (Özdamar 2000: 26).

Nur drei für den Migranten grundlegende Wörter in der Muttersprache werden erinnert: "Arbeiter", "Sehen", "Lebensunfall" (Özdamar 1998: 11f.). Die Grenzen zwischen Eigenem und Fremdem verschwinden, der Referenzpunkt der Vergangenheitsbeurteilung ist allerdings die Gegenwart: "Ich fragte sie auch, warum Istanbul so dunkel geworden ist, sie sagte: 'Istanbul hatte immer diese Lichter, deine Augen sind an Alamanien-Lichter gewöhnt'" (Özdamar 1998: 9). Die Figuren definieren sich jedoch nicht in Antagonismus zu den Deutschen, sondern in Bezug auf die Migrationssituation. 
In Mutterzunge werden durch die Mimikry des Orientalismus Macht und Herrschaftsverhältnisse (in der Politik, zwischen den Geschlechtern) thematisiert. Außerdem verweist Özdamar auf den Gegensatz zwischen westeuropäischer Zivilisation und dem Stereotyp orientalischer Rückständigkeit, ganz im Sinne von E. Saids Buch Orientalismus. Mit der Figur des sprechenden Esels "Karagöz" parodiert Özdamar traditionsorientierte anatolische Denkweisen; durch seine Ironisierung des "Gastarbeiterhandbuchs" entlarvt der Esel die deutsche Arbeitsvermittlungsbehörde in Istanbul als Disziplinierungsinstanz: "Lieber Bruder Arbeiter! Die Toiletten in Europa sind anders als bei uns: wie ein Stuhl. Ihr sollt nicht drauf stehen" (Özdamar 1998: 77).

In den Werken Özdamars dominieren Tropen, die im bildlichen sowie im übertragenen Sinn gebraucht werden; der Grad der Distanz zwischen dem eigentlichen und dem übertragenen Ausdruck ist unterschiedlich, er reicht von der Grenzverschiebungs- zur Sprungtrope ("Hühner" für Studenten): "Die Hühner rauchten, die Hühner gingen ins Kino und schauten Filme" (Özdamar 2000: 157). Auf rhetorische Figuren und Vergleiche trifft man im Werk Özdamars häufig; ein tertium comparationis verbindet die miteinander verglichenen Phänomene:

Sie schauten alle dem Mann zu, der wie eine Eule aussah, und wenn er einen Satz sagte, ging ein anderer in diesen Satz hinein wie eine große Schere, schnitt den Satz in der Mitte durch und vervollständigte den Satz selbst [...]. Ihr Kopf sah aus wie ein Brotlaib, den ein verschlafener Bäckerlehrling gebacken hatte, groß und schief. Sie trug ihn auf den hochgezogenen Schultern wie auf einem Kaffeetablett. (Özdamar 2000: 231 und 11).

Im Titel des Bandes Mutterzunge operiert die Autorin mit der Metonymie "Zunge" für Sprache, und zwar als Umbenennung des eigentlich gemeinten Wortes "Sprache"; sie ist auf der Suche nach der arabischen "Großvater Zunge" und der türkischen "Mutter Zunge"; ihre Sprache ist also nicht ihr Eigentum. Die Autorin spielt auch auf den Begriff "übersetzte Sprache" an, der wörtlich aus dem Türkischen übersetzt "gedrehte Zunge" heißt:

In meiner Sprache heißt Zunge: Sprache./Zunge hat keine Knochen, wohin man sie dreht, dreht sie sich dorthin [...]. Wenn ich nur wüsste, wo ich meine Mutterzunge verloren habe... Ich erinnere mich jetzt an Muttersätze, die sie in ihrer Mutterzunge gesagt hat, nur dann, wenn ich ihre Stimme mir vorstelle, die Sätze selbst kamen in meine Ohren wie eine von mir gut gelernte Fremdsprache. (Özdamar 1998: 9)

Das Türkische fungiert hier im Sinne Genettes als vorangehender "Hypotext" (Genette 1993: 14); die Autorin denkt türkisch und schreibt deutsch, wobei sie grammatische Regeln teilweise ignoriert und ein reduziertes, parataktisches Deutsch wählt, das ihren Status der Fremden widerspiegelt. Dasselbe Verfahren ist in der Metapher "Diamant" für Jungfräulichkeit nachzuweisen (durch den Sprung in eine andere Bildsphäre) oder in der Wendung "beleidigter Bahnhof", wobei die Autorin hier sogar das Ver- 
fahren der Umbenennung erläutert, und zwar über die Polysemie des türkischen Begriffs "zerbrochen" auf der lexikalischen Ebene: "aus dem Busfenster sah ich den Anhalter Bahnhof [...]. Wir nannten ihn den zerbrochenen Bahnhof. Das türkische Wort für 'zerbrochen' bedeutete gleichzeitig auch beleidigt. So hieß er auch 'der beleidigte Bahnhof'" (Özdamar 2000: 25). Ambiguität konstituiert hier die Literarizität von Literatur. Nach Jakobson ist die poetische Funktion definiert als Ausrichtung auf die Sprache selbst, auf formale Eigenschaften des Sprachmaterials; dadurch werden Parallelismen auf phonologischer, syntaktischer und semantischer Ebene erzeugt. Es handelt sich also um "eine besondere Verwendung des sprachlichen Selektionsprinzips. [...] Ein literarischer Text ist dann dadurch ausgezeichnet, dass die in ihm vorhandenen sprachlichen Funktionen von der poetischen Funktion dominiert werden" (Barsch 2001: 376). Verfremdungseffekte bedingen den semantischen Mehrwert innovativer Sprachschöpfungen, so H. Weinrich:

Erinnern wir uns, daß die russischen Formalisten [...] von Poesie nur dann sprechen wollten, wenn die Sprache des literarischen Textes so beschaffen ist, daß sie die Aufmerksamkeit des Lesers wenigstens in Spuren bei den Wörtern festhält, und sie daran hindert, vorschnell zu den Sachen durchzudringen. Eine kunstvoll erschwerte, ja 'verbogene' Sprache, so Sklovskij, ist am besten geeignet, die alltägliche Routine des Lesers aus ihrem eingefahrenen Gleis zu werfen und auf diese Weise jene erwünschte Verlangsamung und Irritation des Rezeptionsvorgangs zu erzwingen, die Voraussetzung für jede Art ästhetischer Erfahrung ist. (Weinrich in Seibert 1984: 58)

Bedeutungsträger und -inhalt fallen bei Özdamar im deutschen Romantext deshalb auseinander, weil sich die Logik des Türkischen dazwischen schiebt. Insofern sind ihre Texte ein Steinbruch literarischer Mehrsprachigkeit: sie verstärkt die der Sprache schon immanente Mehrdeutigkeit dadurch, dass sie ihre beiden Sprachen Türkisch und Deutsch vermischt. Es entstehen für die Struktur der türkischen Wortbildung typische agglutinierende Reihungen von Wörtern (Uygul 1988: 189) sowie Wortneuschöpfungen und -reihungen: "Filmzeigermaschine", "Ostgroßvater", "Schriftzimmer", "Herzstimme", "Geduldstein", "Weingebender", "Schriftzimmerschlüssel”. Mit den türkischen Wörtern unter ihrer Zunge ist Özdamar in den Raum der deutschen Sprache eingedrungen, doch in der "Fremdsprache haben Wörter keine Kindheit" (Özdamar 1998: 44). Die Autorin benutzt ein kreolisiertes, hybrides Deutsch und bildet unter anderem das "Gastarbeiterdeutsch" ab; dabei wechselt sie ständig zwischen den beiden Bedeutungssystemen Deutsch und Türkisch, wobei neue Referenzbezüge entstehen und das Ausdruckspotential ihrer poetischen Sprache erweitert wird. Auch im Roman Die Brücke vom goldenen Horn dominiert das inszenierte Sprechen; Özdamar kritisiert dort ausdrücklich die offizielle türkische Geschichtsschreibung, die demokratische Kulturgeschichte der Türkei betreffend. Auch wird durch Inszenierung einer grammatikalisch verbogenen deutschen Sprache ebenso wie durch das Prinzip der Anthropomorphisierung ein Verfremdungseffekt erzeugt; Özda- 
mar definiert auf der Schauspielschule in Istanbul den Verfremdungseffekt als passionierte Schauspielerin, die dann 1976-77 Brecht-Inszenierungen an der Ostberliner Volksbühne (Özdamar 2003: 103) hautnah miterlebte, noch auf ganz eigenwillige Weise: "'Der Verfremdungseffekt war dagegen für mich wie ein Damoklesschwert [...] Es wurde fast zur Bedrohung, weil wir in der Praxis nicht genau verstanden, was eine Verfremdung sein könnte. [...] Zum Beispiel, wenn ich vom Schiff kam, fragte ich mich, was ist in diesem Moment der Verfremdungseffekt?"' (Özdamar in von Saalfeld 1989: 167).

Das Prinzip der Anthropomorphisierung trägt zur Konkretisierung des Dargestellten bei und entspricht so der "Konkretheit des türkischen Denkstils"', der wahrnehmungsnahe, anschauliche Formulierungen bevorzugt und emphatisches Lesen ermöglicht:

Am nächsten Morgen liefen viele Istanbuler Schuhe im Stadtzentrum über Tintenflecken [...]. Dann flogen die Steine der Polizisten gegen die Fotos des toten Vedat und zerrissen sie in Stücke. [...] Das Schiff kam auf der europäischen Seite an, alle linken, rechten und religiösen Zeitungen falteten sich und wanderten gefaltet in die Taschen der drei Gruppen. Wenn die Zeitungen in den Taschen der Menschen schwiegen, fingen die Istanbuler Mauern an zu sprechen, linke, religiöse und faschistische Slogans. (Özdamar 2000: 260, 261, 294)

Die hybride Sprachform der Migrantin wird zum Ausdruck ihrer spezifischen kulturellen Identität, die von Hybridität geprägt ist; der Zweitsprachenerwerb wird als Prozess der Nachahmung geschildert (Özdamar 2000: 24, 26). Die Strategie der sprachlichen Maskerade und Inszenierung stellt durch das Spiel mit der Hybridität der Migrantensprachen auch das Fremde als Rolle dar, die wieder abgelegt werden kann.

Die Figuren der Werke Özdamars illustrieren Welschs These, dass "Transkulturelle Identitäten [...] eine kosmopolitische Seite ebenso wie eine Seite lokaler Zugehörigkeit" besitzen (Welsch 1997: 71), die sowohl zu einem Verschwimmen der Grenzen, zu Synkretismen, zur Kreolisierung und zum "cross-over" führen. In Özdamars Werk wird uns dies auf subversivironische Weise durch "gespielte Naivität" und das "surreal Anmutende" (Sölcün 2002: 92, 98) vorgeführt; die subversive Mimikry verweist darauf, dass die Nachahmung des Deutschen durch Fremde immer eine Differenz beeinhaltet: "Der Frabrikherr hieß Herr Schering. Sherin sagten die Frauen, Sher sagten sie auch. Dann klebten sie Herr an Sher, so hieß er in manchen Frauenmündern Herschering oder Herscher" (Özdamar 2000: 16). Die Autorin (die auch als Schauspielerin und Theaterregisseurin ausgewiesen ist), setzt Sprache als Signal der hybriden Identität nicht nur bewusst fehlerhaft, sondern als sinnlich-konkrete Reflexion des kommunikativen Spannungsverhältnisses auch betont spielerisch ein. Damit werden sprachliche und identitätsgebundene Probleme der Fremde bewältigt, die sonst als unüberwindbare Dichotomien zur Bewusstseinsspaltung führen könnten; Özdamar gelingt es, ein poetisches Deutsch mit türkischem Zungenschlag zu schreiben. Der Vater der Ich-Erzählerin urteilt nach ihrer Rückkehr nach Istanbul: 
"Sie ist als Nachtigall nach Alamania geflogen und dort ein Papagei geworden, sie hat die deutsche Sprache gelernt. Jetzt ist sie eine türkische Nachtigall und zugleich ein deutscher Papagei" (Özdamar 2000: 157). Damit verweist der Vater auf die grundlegende Rolle der Sprache und der Ethnizität bei der Identitätskonstruktion der Tochter und Migrantin zwischen den Kulturen, aber auch auf ihre Fremdheit in beiden Kulturen. Bei Özdamars Darstellung der Migrationssituation handelt es sich weder um Betroffenheitsliteratur noch um Sozialreportagen; es werden jedoch die Ursachen für die Migration von Frauen aus der Türkei, die Lebenswirklichkeit im Wohnheim für türkische Gastarbeiterinnen und vor allem die Migrationsmotive der Ich-Erzählerin fokussiert. Ihr Bewusstwerdungs- und Emanzipationsprozess bildet das Gerüst des Romans. Im ersten Teil geht es vor allem um die Zerreißprobe zwischen den Rollenmustern des Herkunftslandes und der neuen Berliner Umgebung mit ihren Freiheiten; es kommt dann zu einem Reifungs- und Entscheidungsprozess, parallel zum sexuellen Erwachen entwickelt sich das politische Bewusstsein der jungen Migrantin. In Özdamars assoziativem Erzählen greift das erzählende Ich weder organisierend noch deutend ein; die Struktur des Romans ist geprägt von Sprunghaftigkeit (was sich im häufigen Wechsel zwischen den Zeitebenen niederschlägt). Diese Sprunghaftigkeit dominiert gerade zu Beginn des Romans (Özdamar 2000: 11-14) im in-medias-res-Romananfang (Broteinkauf), des Weiteren in der Rückblende in die Istanbuler Jugend am Theater (Özdamar 2000: 12-13), dann im Rückgriff auf den chronologisch exakten Romananfang (Özdamar 2000: 14-17), wobei die Handlung aus einer Aneinanderreihung von Szenen besteht (die Zugfahrt von Istanbul nach Berlin, die Erlebnisse im Wohnheim, in Berlin und bei der Arbeit im Telefunken-Werk, dann im Flugzeug von München nach Berlin und wieder im Wohnheim). Weitere Ortswechsel sind: Istanbul-Berlin-Istanbul (Özdamar 2000: 11, 106); Bodensee-Berlin (Özdamar 2000: 108, 109); HannoverParis (Özdamar 2000: 124); Berlin-Istanbul (Özdamar 2000: 146, 170). Diskontinuität entsteht auch über häufige Erinnerungen an die Türkei in Berlin und an Berlin in der Türkei (Özdamar 2000: 50, 176, 193). Auch die verschiedenen Männer, die im Leben der Ich-Erzählerin eine Rolle spielen, bilden ein Element der Diskontinuität: der Heimleiter (Özdamar 2000: 72); der Student vom Sozialistischen Studentenbund; Jordi; der "hinkende Sozialist"; "der Intellektuelle, der wie eine Eule aussah"; Kerim; "ein paar Jungen aus der Schauspielschule” (Özdamar 2000: 31, 28, 151, 166, 233, 251, 210).

Andererseits wird die Handlung zusammengehalten von Parallelstrukturen, d.h. von semantischen Strukturen der Kontinuität und der Rekurrenz verschiedenster Inhalts- und Ausdruckselemente im Sinne einer verbindenden Isotopieebene. Faktoren der Kontinuität im Leben der IchErzählerin sind: die Schauspielerei, das starke Interesse am Kommunismus, das Rauchen und die um den Verlust des "Diamanten" kreisenden Jungfernhautgeschichten (Özdamar 2000: 193). Verknüpfungen werden hergestellt durch Leitmotive wie die "Bluse" als Erinnerung an Berlin (Özdamar 2000: 176, 234) und durch Jordi, der durch Gedanken und Briefe immer 
wieder evoziert wird (Özdamar 2000: 224, 323). Der Romantext erweist sich also als hybride Textfläche, in der nicht-sukzessiv angeordnete Handlungslogik zu simultaner Artikulation von Erinnerungen geworden ist. Özdamar hat performativ auf der Ausdrucksebene eine Repräsentationsform gefunden, "die die eigentümliche Verfaßtheit der modernen Welt in ihren ontologischen Strukturen wiedergeben und so eine Semantik für den Umgang mit polykontexturalen Umwelten begründen" (Bronfen \& Marius 1997: 28) kann. Schreiben wird bei Özdamar zu einem Nachvollzug des Migrantendaseins, des Sich-Treiben-Lassens im Wort, denn es eröffnet "einen Raum, der zu Bewegung einlädt, zu Migration, zu einer Reise"; nur im Schreiben gelingt es, eine gewisse Distanz aufzubauen "zwischen uns selbst und den Kontexten, die unsere Identität definieren", denn "wie das mehrdeutige Reisen beginnt es mit bekannten Materialien [...] und versucht doch aus den Grenzen seiner Bewegung, aus der Erfahrung des Ortswechsels ein Mehr herauszuholen, einen Überschuß" (Chambers 1996: 11, 12).

\section{Fazit}

Das emanzipatorische Potential der Werke von Özdamar und Zaptcioglu liegt in der Revalorisierung der kulturellen Hybridität postkolonialer "borderline conditions", in denen soziale, kulturelle und geschlechtsspezifische Gegensätze abgebaut werden. Hybridität steht im Gegensatz zu Vorstellungen von Einheitlichkeit und Eindeutigkeit, von Hegemonie und machtgestütztem Monolog. In der Postmoderne wird Hybridität als Gegenbegriff zum Hierarchischen, Hegemonialen und Monologischen verstanden. In Abgrenzung sowohl vom Konzept der Einzelkulturen als auch von dem der Multikulturalität entwickeln beide Autorinnen Welschs Begriff von Transkulturalität weiter, denn die Beschreibung heutiger Kulturen als Inseln ist deskriptiv falsch und normativ irreführend. Unsere Kulturen haben vielmehr eine neue Form angenommen, die man als transkulturell bezeichnen kann, weil sie durch die traditionellen Kulturgrenzen wie selbstverständlich hindurchgehen. Wie die beiden Autorinnen in ihren Werken zeigen, sind die kulturellen Verhältnisse heute weithin durch Mischungen und Durchdringungen gekennzeichnet. Transkulturalität ist somit eine Folge der inneren Differenzierung und Komplexität der modernen, von Migration geprägten Kulturen. Diese umfassen eine Vielzahl von Lebensformen und Sprachen, die einander durchdringen oder auseinander hervorgehen können.

\section{Bibliographie}

Ackermann, Ingrid (1985). "Integrationsvorstellungen und Integrationsdarstellungen in der Ausländerliteratur." Informationen Deutsch als Fremdsprache 12(3), 222-232.

Adelson, Leslie (1991). “Migrantenliteratur oder deutsche Literatur?” P.M. Lützeler, 
(Hrsg.) (1991). Spätmoderne und Postmoderne. Beiträge zur deutschen Gegenwartsliteratur. Frankfurt am Main: Fischer, 67-81.

Amodeo, Immacolata (1996). Die Heimat heißt Babylon: Zur Literatur ausländischer Autoren in der Bundesrepublik Deutschland. Opladen: Westdeutscher Verlag.

Barsch, Achim (2001). "Literarizität.” A. Nünning (Hrsg.) (2001). Metzler Lexikon Literatur- und Kulturtheorie. Ansätze-Personen-Grundbegriffe. Stuttgart/ Weimar: Metzler, 376-377.

Blioumi, Aglaia (Hrsg.) (2002). Migration und Interkulturaliät in neueren literarischen Texten. München: Iudicium.

Borrelli, Michele \& Gerd Hoff (Hrsg.) (1988). Interkulturelle Pädagogik im internationalen Vergleich. Hohengehren: Burgbücherei Schneider.

Briegleb, Klaus \& Sigrid Weigel (Hrsg.) (1992). Gegenwartsliteratur seit 1968. Hansers Sozialgeschichte der deutschen Literatur vom 16. Jahrhundert bis zur Gegenwart. München/Wien: Hanser.

Bronfen, Elisabeth, Marius, Benjamin \& Therese Steffen (Hrsg.) (1997). Hybride Kulturen. Beiträge zur angloamerikanischen Multikulturalismus-Debatte. Tübingen: Stauffenberg.

Bhabha, Homi K. (1996). "Postkoloniale Kritik. Vom Überleben der Kultur." Das Argument 215, 345-361.

Bhabha, Homi K. (2000). Die Verortung der Kultur. Tübingen: Stauffenburg.

Biermann, Heinrich \& Bernd Schurf (Hrsg.) (1999). Texte, Themen und Strukturen. Deutschbuch für die Oberstufe. Berlin: Cornelsen, 360-365.

Chambers, Iain (1996). Migration, Kultur, Identität. Tübingen: Stauffenburg.

Chiellino, Carmine (Hrsg.) (2000). Interkulturelle Literatur in Deutschland. Stuttgart: Metzler, 135-153.

Esselborn, Karl (1997). "Von der Gastarbeiterliteratur zur Literatur der Interkulturalität." Jahrbuch Deutsch als Fremdsprache 23, 47-77.

Gates, Henry Louis (1988). Signifying Monkey. Oxford: Oxford University Press.

Genette, Gérard (1993). Palimpseste: Die Literatur auf zweiter Stufe. Frankfurt am Main: Suhrkamp.

Howard, Mary (1997). "Einleitung." Howard, Mary (Hrsg.) (1997). Interkulturelle Konfigurationen: Zur deutschsprachigen Erzählliteratur von Autoren nichtdeutscher Herkunft. München: Iudicium, 7-17.

http://www.kanak-attack.de/html/presse/welt-interview.htm (Zugriff: 15.5. 03).

Lützeler, Paul Michael (Hrsg.) (1991). Spätmoderne und Postmoderne. Beiträge zur deutschen Gegenwartsliteratur. Frankfurt am Main: Fischer.

Nünning, Ansgar (Hrsg.) (2001). Metzler Lexikon Literatur- und Kulturtheorie. Ansätze-Personen-Grundbegriffe. Stuttgart/Weimar: Metzler.

Özdamar, Emine S. (1998). Mutterzunge. Köln: Kiepenheuer.

Özdamar, Emine S. (2000). Die Brücke vom goldenen Horn. Köln: Kiepenheuer.

Özdamar, Emine S. (2003). Seltsame Sterne starren zur Erde. Köln: Kiepenheuer.

Rösch, Heidi (1992). Migrationsliteratur im interkulturellen Kontext. Frankfurt: Verlag für interkulturelle Kommunikation.

Schneider, Irmela \& Christian W. Thomsen (Hrsg.) (1997). Hybridkultur. Medien, Netze, Künste. Köln: Wienand.

Seibert, Peter (1984). “Zur 'Rettung der Zungen'.” Lili 14 (56), 40-62.

Sölcün, Sargut (2000). "Literatur der türkischen Minderheit.” C. Chiellino (Hrsg.) (2000). Interkulturelle Literatur in Deutschland. Stuttgart: Metzler, 135-153.

Sölcün, Sargut (2002). "Gespielte Naivität und ernsthafte Sinnlichkeit der Selbstbegegnung - Inszenierungen des Unterwegsseins in Emine Sevgi Özdamars Roman Die Brücke vom Goldenen Horn.” A. Blioumi (Hrsg.) 
(2002). Migration und Interkulturaliät in neueren literarischen Texten. München: Iudicium, 92-112.

Uygul, Nermi (1988). "Türkisch-Deutsch. Eine transkulturelle Betrachtung." M. Borrelli \& G. Hoff (Hrsg.) (1988). Interkulturelle Pädagogik im internationalen Vergleich. Hohengehren: Burgbücherei Schneider, 189-197.

von Saalfeld, Lerke (Hrsg.) (1989). Ich habe eine fremde Sprache gewählt. Gerlingen: Bleicher.

Weigel, Sigrid (1992). "Literatur der Fremde - Literatur in der Fremde." K. Briegleb \& S. Weigel (Hrsg.) (1992). Gegenwartsliteratur seit 1968. Hansers Sozialgeschichte der deutschen Literatur vom 16. Jahrhundert bis zur Gegenwart. München/Wien: Hanser, 182-229.

Wägenbaur, Thomas (1995). "Kulturelle Identität oder Hybridität?” LiLi 25 (97), 22-47.

Weinrich, Harald (1984). "Gastarbeiterliteratur in der Bundesrepublik Deutschland." LiLi 14 (56), 22-32.

Welsch, Wolfgang (1997). "Transkulturalität. Zur veränderten Verfassung heutiger Kulturen.” I. Schneider \& C. W. Thomsen (Hrsg.) (1997). Hybridkultur. Medien, Netze, Künste. Köln: Wienand, 67-90.

Wierschke, Annette (1996): Schreiben als Selbstbehauptung: Kulturkonflikt und Identität in den Werken von Aysel Özakin, Alev Tekinay und Emine Sevgi Özdamar. Frankfurt: Verlag für interkulturelle Kommunikation.

Zaptcioglu, Dilek (1998). Der Mond isst die Sterne auf . Stuttgart/Wien/Berlin: Thienemann.

${ }^{1}$ Der Autor F. Zaimoglu zählt sich etwa zur "größten deutschen Minderheit auf deutschem Boden, den Türkisch- und Kurdischstämmigen der zweiten Generation." http://www.kanak-attack.de/html/presse/welt-interview.htm

${ }^{2}$ U.a. ein Auszug aus Mutterzunge von Özdamar und Gedichte von Franco Biondi.

${ }^{3}$ Vgl. Ugyul (1988: 190). Weitere Merkmale der türkischen Sprache, die als Subtext für Özdamar prägend ist: Redensarten haben ihren Ursprung in Körperteilen wie Kopf, Hand, Fuß; die Namensgebung für Farben lässt eine detaillierte Farbbeschreibung zu; häufige Personifizierung statt der im Deutschen üblichen Impersonalia ("gec vakit simsekler cakti=zur späten Zeit blitzten Blitze, statt dt.: später hat es geblitzt"); der Bezug zur subjektiven Welt, zur "inneren Wirklichkeit" ist im Türkischen vordergründig; die Denkrichtung ist von Innen nach Außen gerichtet ("seni gönlümden cikaramam=ich kann dich nicht aus meinem Gemüt wegschaffen; dt.: ich kann dich nicht vergessen"). 